EUROPEAN ORGANIZATION FOR NUCLEAR RESEARCH

CERN - AB Department

CERN-AB-2007-033

\title{
WAKEFIELD MODELS FOR PARTICLE TRACKING CODES
}

\author{
A. Latina, R. J. Barlow ${ }^{3)}$, G. A. Blair ${ }^{1)}$, A. Bungau ${ }^{3)}$, S. Malton ${ }^{1)}$, G. Rumolo, D. Schulte, J.D.A. Smith ${ }^{2)}$ \\ CERN, Geneva, Switzerland
}

\begin{abstract}
Wakefields have a considerable effect on beam dynamics and they must not be neglected for emittance growth studies, background estimates and other problems. The codes used for these problems are normally not capable of self-consistent wakefield calculations. They should thus be extended with either analytical models or export the wakefields numerically evaluated with other codes when analytical models are not feasible. We discuss both approaches and present their implementation in PLACET and BDSIM. The simulation results for the ILC and CLIC beam delivery systems are given as an example.
\end{abstract}

\footnotetext{
${ }^{1)}$ Royal Holloway, University of London, Surrey

${ }^{2)}$ Lancaster University, Lancaster

3) UMAN Manchester
}

Presented at

PAC07, 22nd PAC Conference, June 25-29, 2007, Albuquerque, USA

Geneva, Switzerland

August 2007 


\title{
WAKEFIELD MODELS FOR PARTICLE TRACKING CODES*
}

\author{
A. Latina, G. Rumolo, D. Schulte (CERN, Geneva), \\ G. A. Blair, S. Malton (Royal Holloway, University of London, Surrey), \\ J.D.A. Smith (Lancaster University, Lancaster), R. J. Barlow, A. Bungau (UMAN, Manchester)
}

\section{Abstract}

Wakefields have a considerable effect on beam dynamics and they must not be neglected for emittance growth studies, background estimates and other problems. The codes used for these problems are normally not capable of self-consistent wakefield calculations. They should thus be extended with either analytical models or export the wakefields numerically evaluated with other codes (such as GdfidL[1]) when analytical models are not feasible. Both approaches are presented as well as their implementation in PLACET[2], MERLIN[3] and BDSIM[4]. The simulation results for the ILC and CLIC beam delivery systems are given as an example. Results produced with different codes are compared.

\section{COLLIMATOR WAKEFIELDS IN PLACET}

In PLACET, to account for the flat structure of a collimator, dipole and quadrupole components of the wake field are weighed by the Yokoya coefficients [5] at the moment when the wake field kick is applied to each bunch particle:

$$
\begin{aligned}
& W_{1 x}^{\perp}\left(x, x_{0} ; z\right)=W_{1}^{\perp}(z) \frac{\pi^{2}}{12}\left(x_{0}+0.5 x\right) \\
& W_{1 y}^{\perp}\left(y, y_{0} ; z\right)=W_{1}^{\perp}(z) \frac{\pi^{2}}{24}\left(y_{0}-y\right)
\end{aligned}
$$

This is true only in the linear regime, that is for bunch particles that stay sufficiently close to the machine axis and far enough from the collimator walls. For halo particles close to the collimator jaws, the nonlinear components of the wake may become significant. The wakes scale as function of the coordinates of source and probe particles[6, 7] (at least in the plane where the gap is smaller) according to:

$$
\begin{gathered}
W_{1 x}^{\perp}\left(x, x_{0}, y, y_{0} ; z\right)=W_{1}^{\perp}(z) \frac{\pi}{8 g}\left[\frac{x_{+} \cos x_{+}+\sin x_{+}}{\cosh y_{-}+\cos x_{+}}+\right. \\
+\frac{\sin x_{+}\left(x_{+} \sin x_{+}-y_{-} \sinh y_{-}\right)}{\left(\cosh y_{-}+\cos x_{+}\right)^{2}}+ \\
\left.\frac{x_{-} \cos x_{-}+\sin x_{-}}{\cosh y_{-}-\cos x_{-}}-\frac{\sin x_{-}\left(x_{-} \sin x_{-}+y_{-} \sinh y_{-}\right)}{\left(\cosh y_{-} \cos x_{-}\right)^{2}}\right] \\
W_{1 y}^{\perp}\left(x, x_{0}, y, y_{0} ; z\right)=W_{1}^{\perp}(z) \frac{\pi}{8 g}\left[-\frac{y_{-} \cosh y_{-}+\sinh y_{-}}{\cosh y_{-}+\cos x_{+}}\right. \\
-\frac{\sin y_{-}\left(x_{+} \sin x_{+}-y_{-} \sinh y_{-}\right)}{\left(\cosh y_{-}+\cos x_{+}\right)^{2}}
\end{gathered}
$$

* This work is supported by the Commission of the European Communities under the $6^{\text {th }}$ Framework Programme "Structuring the European Research Area", contract number RIDS-011899.

$$
\begin{gathered}
\frac{y_{-} \cosh y_{-}+\sinh y_{-}}{\cosh y_{-}-\cos x_{-}} \\
\left.\frac{\sinh y_{-}\left(y_{-} \sinh y_{-}+x_{-} \sin x_{-}\right)}{\left(\cosh y_{-}-\cos x_{-}\right)^{2}}\right]
\end{gathered}
$$

with

$$
x_{+}=\frac{\pi}{2 g}\left(x+x_{0}\right), x_{-}=\frac{\pi}{2 g}\left(x-x_{0}\right), y_{-}=\frac{\pi}{2 g}\left(y-y_{0}\right)
$$

The first of the above equations reduces to:

$$
\begin{gathered}
W_{1 x}^{\perp}\left(x, x_{0} ; z\right)= \\
=W_{1}^{\perp}(z) \frac{\pi}{8 g}\left(\frac{x_{+}+\sin x_{+}}{1+\cos x_{+}}-\frac{x_{-}-\sin x_{-}}{1-\cos x_{-}}\right)
\end{gathered}
$$

if the beam lies vertically close to the plane $y=0$ such that $\left(y, y_{0}\right) \ll g$, or in general when $y-y_{0} \ll g$. The above formulæ are general and account for the near-wall effects. These formulæ have been implemented in PLACET. A compromise between a requirement for low noise tracking and computational speed is certainly necessary for this type of calculations. For example, the non-oscillatory character of the wake allows for a coarse slicing of the bunch (even if the initial drop needs to be adequately resolved), which also translates into a lower number of macroparticles required to model the bunch. The difference between linear and nonlinear coefficients is shown in Fig. 1 for the "on axis" case $y=y_{0}$, Eq.4. At about half the gap size the wake becomes significantly nonlinear with the coordinates of the source and probe particles and increases more steeply going toward the chamber wall. Nevertheless, as $y$ starts differing from $y_{0}$ significantly in units of $g$, the geometric cross terms in Eq. 2, which make the horizontal wake depend on the vertical coordinates and vice versa, cause a decrease of the effective wake values.

Figures 2 shows how the nonlinear wake field changes when going off axis: curves at $y-y_{0}=0.5 \mathrm{~g}$ (blue) and $y-y_{0}=0.8 g$ (red) are plotted, which clearly show how the effect of the wake, amplified on axis by the nonlinear contribution, becomes quickly weaker when moving away from the plane $y=y_{0}$

\section{COLLIMATOR WAKEFIELDS IN MERLIN}

The higher order wakefield modes are implemented into the MERLIN code as the they become important when the bunch passes through a collimator close to the collimator wedges. The collimator do not have an axial symmetry as the system is not characterized by resonances.

The formalism for incorporating intra-bunch wakefields into the particle tracking code MERLIN starts with several assumptions: 

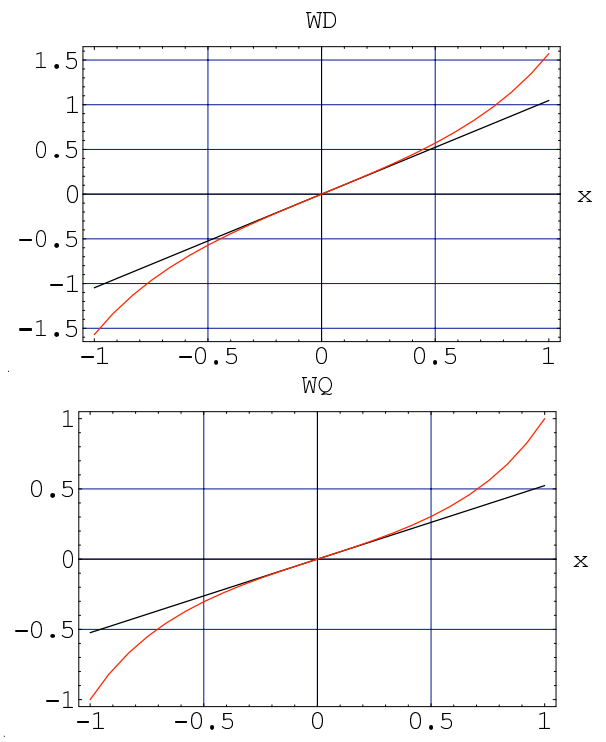

Figure 1: Dipole (left) and quadrupole (right) components of the wake field as a function of $x_{0} / g, x=0$ and $x_{0}=0, x / g$ respectively, for $y, y_{0} \ll g$. The fully nonlinear expressions (red lines) are compared with their linear approximations for $x_{0}, x \ll$ $g$ (black lines).

- all particles are relativistic ( $v \approx c, \gamma$ large); therefore the effect of the charges on each other is suppressed by a power of $\gamma$ and therefore ignored.

- the effects of transverse velocity and acceleration are ignored (the position $r$ and $r^{\prime}$ of the leading and trailing particle are constant)

- the collimator aperture is assumed to be circular (the half width of the collimator is treated as radius in our model)

The particle bunches are distorted from their original Gaussian shape during passage, therefore trigonometric terms are important to describe the internal structure on the same scale as the beam pipe. If we consider the effect on a trailing particle at $r, \theta$ of a slice of $N$ particles all ahead by the same distance $s$ (their parameters are $r^{\prime}$ and $\theta^{\prime}$ ), the total effect is given by simple summation. Writing

$C_{m}=\sum r^{\prime} m \cos \left(m q^{\prime}\right)$, and $S_{m}=\sum r^{\prime} m \sin \left(m q^{\prime}\right)$,

where the summation is over all particles in a slide, then the combined kick is:

$$
\begin{aligned}
W_{z}= & \sum W_{m}^{\prime}(s) r^{m}\left(C_{m} \cos (m \theta)+S_{m} \sin (m \theta)\right) \\
W_{x}= & \sum m W_{m}(s) r^{m-1}\left(C_{m} \cos ((m-1) \theta)+\right. \\
& \left.+S_{m} \sin ((m-1) \theta)\right) \\
W_{y}= & \sum m W_{m}(s) r^{m-1}\left(S_{m} \cos ((m-1) \theta)+\right. \\
& \left.+C_{m} \sin ((m-1) \theta)\right)
\end{aligned}
$$

The usual monopole and dipole formulæ are reproduced for $m=0,1$. For a particle in a slice $i$, a wakefield effect is received for all slices $j \geq i$. So the total effect for the $x$ direction for example is:

$$
\sum_{j} w_{x}=\sum_{m} m r^{m-1} \cdot\left(\cos ((m-1) q) \sum_{j} W_{m}\left(s_{j}\right) C_{m j}+\right.
$$

$$
\left.+\sin ((m-1) q) \sum_{j} W_{m}\left(s_{j}\right) S_{m j}\right)
$$

The sums $C_{m}$ and $S_{m}$ depend only on the leading slice, $W_{m}$ depends only on the aperture geometry and $r$ and $\theta$ depend only on the trailing particle. Fig. 3 shows an example of high order mode wakefields calculated by MERLIN.
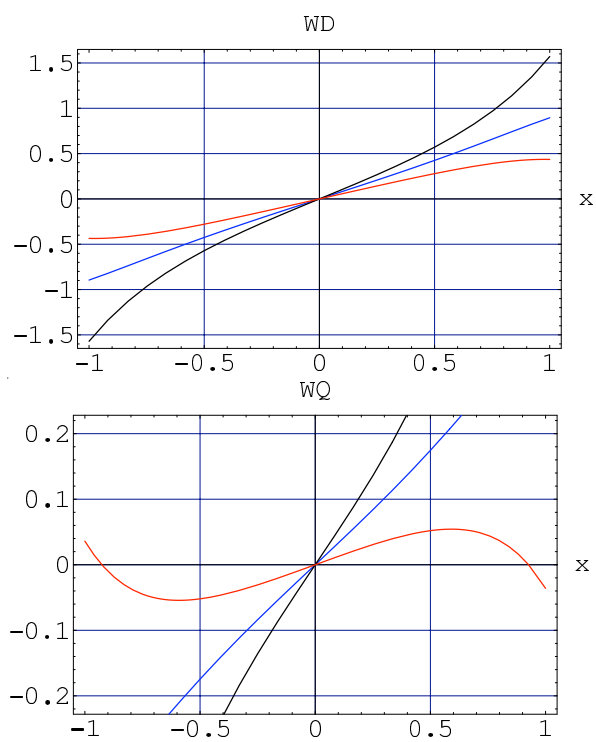

Figure 2: Dipole (left) and quadrupole (right) components of the wake field as a function of $x_{0} / g, x=0$ and $x_{0}=0, x / g$ respectively, for $\left(y-y_{0}\right) / g=0$ (black), 0.5 (blue), 0.8 (red).

\section{PLACET-BDSIM INTERFACE}

Halo particles which are close to the walls of the beampipe may be kicked by the collimator wakefields and interact with the beampipe material, producing secondary particles. A single simulation code that implements both tracking and secondary particles generation, does not exist. On the one hand, codes designed to track single particles and their secondaries deriving from the interactions with the materials, not including intra-bunch interactions, exist (BDSIM); on the other hand, codes such as PLACET and MERLIN take into account collective effects but do not simulate the interactions of the particles with the walls of the beamline.

Combining the abilities of BDSIM and PLACET allows to achieve an accurate simulation of the secondary particles generation and their tracking in such delicate components as the collimators, taking into account the most relevant collective effects. One method to achieve this is under development. It consists in interfacing the PLACET tracking code and BDSIM, based on the following idea: the tracking is performed in parallel by both codes. BDSIM tracks core and halo particles, while PLACET tracks only the core particles. When BDSIM reaches an element where it is desired to include the effects of wakefields, BDSIM sends to PLACET the halo particles to perform the calculations of the wakefield kick; then, PLACET reports the resulting 
kick angles for each particle back to BDSIM, which applies the kicks and continues the tracking.

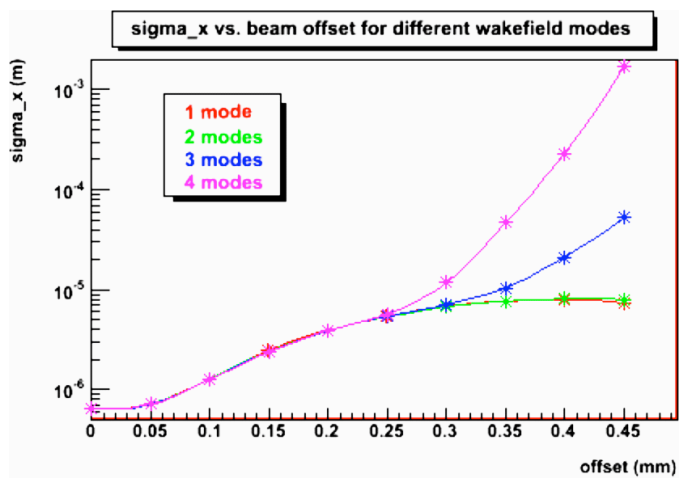

Figure 3: The increase of the horizontal beam size due to the high order mode wakefields as calculated by MERLIN.

Although the exchange of particle information has been successfully implemented, difficulties are currently being experienced in the post-exchange tracking. The work is still in progress.

\section{IMPORTING NUMERICAL WAKEFIELDS}

When analytical models are not feasible, the wakepotentials should be calculated using numerical solvers, such as GdfidL. The wakepotentials calculated with such codes should thus be imported into the tracking codes, in order to be used during the tracking.

Even exploiting the intrinsic symmetries of the wakepotentials, the wakefunctions calculated using these solvers may require a considerable amount of disk space if the full information of a computation in a fine mesh is requested. Depending on the collimator's geometry and on the beam size, they can even require several gigabytes of disk storage per collimator. Such big files are not very practical and they need to be compressed in order to be actually used into tracking codes.

In order to compress the wakepotential files, a code was written to read the output files produced by GdfidL and perform the multipole expansion of the wakepotentials in there, taking into account the symmetries of the collimator to further reduce the number of coefficients that need to be stored. In order to reproduce a typical longitudinal wakepotential generated in a rectangular collimator, only few modes are usually necessary and therefore the final output file requires only a few kilobytes on disk. Fig. 4 show a longitudinal wake potential calculated using GdfidL and its multipole expansion up to the 5-th (decapole) mode, in the region of convergence. For such expansion only 5 complex numbers need to be stored on disk, instead of a grid of numbers mapping the potential in the whole collimator space as the direct output of the solver is. To cover a whole threedimensional volume, the collimator is divided in slices and the multipole expansion is performed slice by slice, storing the results into a single output file.

Another code, that soon will be included into PLACET, is already capable of to reading the multipole expansion of a longitudinal wakepotential and restoring the information in the whole three-dimensional volume of the collimator. This code also calculates the transverse components of the wakepotential using a numerical implementation of the Panofsky-Wenzel theorem and finally returns the wakefield kick at any position in the volume.

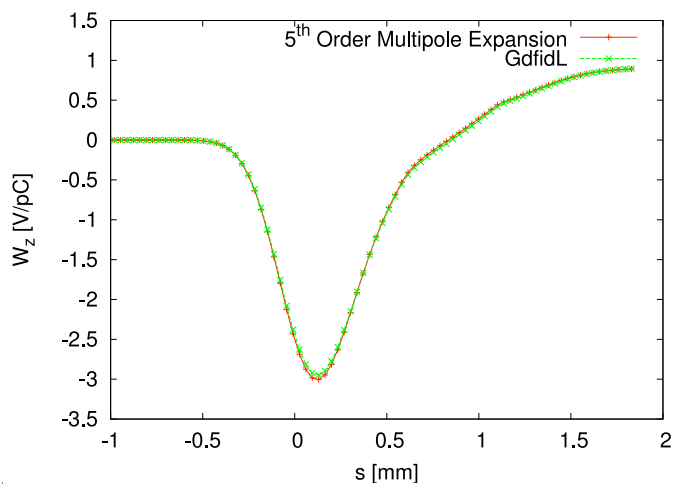

Figure 4: Longitudinal component of the wake-potential generated by a test charge in a simple test geometry, as calculated using GdfidL (green) and reconstructed via a multipole expansion to the $5^{\text {th }}$ order (red). In this case, the files produced using GdfidL required $40 \mathrm{Mb}$ of disk space, whereas the multipole coefficients used less than $10 \mathrm{~Kb}$.

\section{CONCLUSIONS}

The collimator wakefields might have a strong impact on the luminosity of future linear colliders. Realistic simulations of the collimators in the ILC and CLIC BDS are being pursued. Analytic calculations of the wakefields are already implemented both in PLACET and in MERLIN. Where analytical methods are not sufficiently accurate, numerical calculations of the wakefields must be carried out using solvers, such as GdfidL, and plugged into the tracking codes. A software package that expands in multipoles and calculates the wakefield kick directly from the solver output has been developed. Files for realistic ILC collimators are already available[8] and it is anticipated that these will shortly be used in tracking calculations of the BDS.

\section{REFERENCES}

[1] http://www.gdfidl.de/

[2] http://savannah.cern.ch/projects/placet

[3] "Merlin - A C++ Class Library for Accelerator Simulations", http://www.desy.de/ merlin

[4] I. Agapov et al., "The BDSIM Toolkit", 2006, EUROTeVReport-2006-014

[5] K. Yokoya, Part. Acc. 41, 221 (1993)

[6] A. Piwinski, DESY 94-068

[7] K. Bane, I. Irwin, T. Raubenheimer, in NLC ZDR, SLAC Report 474, Vol. 2, p. 570 (1996)

[8] J.D.A. Smith, C. Glasman, "Computation of Wakefields in the ILC Collimators", this conference 